Advances in Applied Physics, Vol. 1, 2013, no. 1, 47 - 58

HIKARI Ltd, www.m-hikari.com

\title{
Relativistic Resonant Tunneling Lifetime for Double Barrier System
}

\author{
S. P. Bhattacharya \\ Department of Physics and Technophysics \\ Vidyasagar University Midnapore 7211 101, India \\ P. K. Mahapatra \\ Department of Physics and Technophysics \\ Vidyasagar University Midnapore 7211 101, India \\ West Bengal Technological University \\ BF - 142, Sector - 1, Salt Lake City, Kolkata 700 064, India
}

\section{Arif Khan*}

Department of Physics, Neotia Institute of Technology managment \& Science

Diamond Harbour Road, Amira 743 368, 24 PGS(S), India

Electrocom Corporation, PO Box: 60317, Potomac, MD 20859, USA

Sciencotech, LLC, 780 Girard Street NW, Washington DC 20001, USA

*a.khan123@yahoo.com

Copyright (C) 2013 S. P. Bhattacharya et al. This is an open access article distributed under the Creative Commons Attribution License, which permits unrestricted use, distribution, and reproduction in any medium, provided the original work is properly cited.

\begin{abstract}
A relativistic study on lifetime of resonant tunneling states in the case of the double barrier system consisting of rectangular potential barriers has been studied. This study reveals the fact that as compared to the non-relativistic resonant tunneling states the relativistic resonant states occur in the lower energies but they are having shorter lifetime..
\end{abstract}

Keywords: Transmission Coefficient, Resonant Tunneling, Resonant Tunneling Lifetime, Double Barrier System, Relativistic Impact 


\section{Introduction}

The electronic properties in condensed matter having heavy atoms require to be treated on relativistic footings. The electrons of such atoms may acquire velocities of the order $10^{8} \mathrm{~cm} / \mathrm{s}$ or more which are only two orders of magnitude smaller than the velocity of light in vacuum. So we may, as a matter of principle, expect relativistic impact on the electronic motion in case of such heavy atoms. Many researchers have been engaging in exploring the relativistic effect on the electronic properties. So far, the following aspects of condensed matter have received the relativistic treatment: (i) bulk states, (ii) surface states, (iii) interface states, and (iv) impurity states.

During last two decades or so, the resonant tunneling has become an important facet of condensed matter physics $[1,2,3,4,5,6,7,8,9]$. In the recent years the investigation of the lifetime of states corresponding to resonant tunneling has drawn considerable attentions $[1,2,3,5,6,7,8]$. While studying the resonant tunneling relativistically, it has been found that the resonant states occur in lower energies as compared to the non-relativistic (NR) ones [10,11]. But as per our knowledge, no investigation regarding the lifetime corresponding to the relativistic resonant tunneling sates has been carried out so far. The purpose of the present study is to report a relativistic treatment in this direction. Briefly, our objectives are to (i) achieve a relativistic expression of the lifetime of the relativistic sates corresponding to resonant tunneling for a double barrier system (DBS), (ii) compare critically the relativistic results with $\mathrm{NR}$ results previously obtained [1, 2, 3, 4, 5, 6], and (iii) elucidate the quantitative impacts of relativistic treatment on the lifetime of the resonant tunneling states.

\section{Model}

Our model, shown in Fig. 1, consists of two rectangular potential barriers of same height separated by a zero potential region. The widths of the barriers and the zero potential region are $b$ and $a$ respectively and the height of the barrier is $V$.

\section{Some aspects of one dimensional time-independent Dirac equation}

The DBS as shown in Fig. 1 is one dimensional (1D) and the motion of the electron is confined along $x$-direction only. In view of this fact, the 1D time-independent Dirac equation becomes worthwhile to carry out the studies of lifetime of the resonant tunneling. The 1D time-independent Dirac equation for an electron moving along $x$-direction in a potential $V(x)$ appears as:

$$
\left[-i \hbar c \sigma_{x} \frac{d}{d x}+m c^{2} \sigma_{z}+V(x)\right] \phi=E_{R} \phi
$$




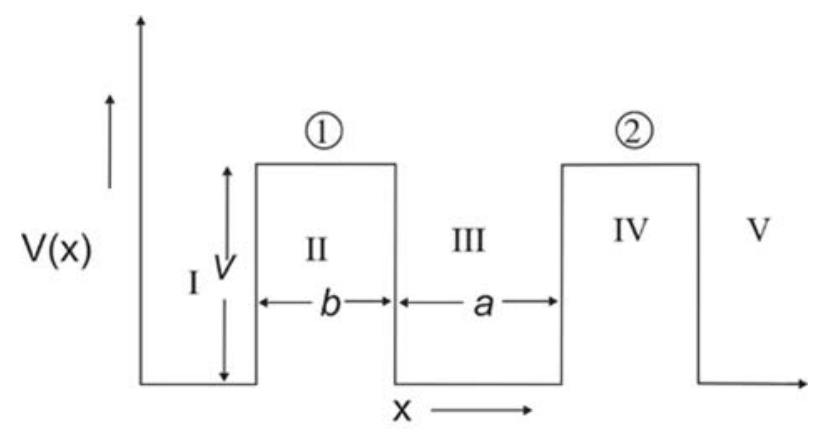

Figure 1: Double barrier system with rectangular barrier type potentials

where $m, c, x$, and $\sigma_{z}$ are the rest mass of the electron, speed of light in vacuum, $x$ and $z$-component of the Pauli spin matrix respectively. The relativistic probability density $(P)$ and the relativistic current density $(J)$ corresponding to Eq. (1) appear as:

$$
\begin{gathered}
P=\phi^{\dagger} \phi \\
J=c \phi^{\dagger} \sigma_{x} \phi
\end{gathered}
$$

The model of our system as shown in Fig. 1 is concerned with the regions of constant potentials. As a result, we would require subsequently the following solution $[10,11]$ of Eq. (1) with a constant potential.

$$
\begin{gathered}
\phi=\left[\begin{array}{l}
1 \\
\gamma
\end{array}\right] p \exp \left(i \rho_{V} x\right)+\left[\begin{array}{c}
1 \\
-\gamma
\end{array}\right] q \exp \left(-i \rho_{V} x\right) \\
\rho_{V}^{2}=\frac{(\epsilon-V)\left(\epsilon-V+2 m c^{2}\right)}{c^{2} \hbar^{2}} \\
\epsilon=E_{R}-m c^{2}
\end{gathered}
$$

It is easy to see that $\epsilon$ is the relativistic kinetic energy of the electron. The advantage of introducing $\epsilon$ lies in fact that it is the relativistic analogue of the NR energy $(E)$ of resonant tunneling states. As we will see later the lifetime of relativistic resonant tunneling states also becomes associated with $\epsilon$. Conforming to the spinor given by Eq. (4), we can write the following forms of spinors in various regions of our DBS:

$$
\begin{aligned}
& \phi_{1}=\text { Spinor in region I } \\
& \qquad=\left[\begin{array}{l}
1 \\
\gamma
\end{array}\right] p_{1} \exp (i \rho x)+\left[\begin{array}{c}
1 \\
-\gamma
\end{array}\right] q_{1} \exp (-i \rho x)
\end{aligned}
$$


$\phi_{2}=$ Spinor in region II

$$
=\left[\begin{array}{c}
1 \\
\gamma^{\prime}
\end{array}\right] p_{2} \exp \left(i \rho^{\prime} x\right)+\left[\begin{array}{c}
1 \\
-\gamma^{\prime}
\end{array}\right] q_{2} \exp \left(-i \rho^{\prime} x\right)
$$

$\phi_{3}=$ Spinor in region III

$$
=\left[\begin{array}{l}
1 \\
\gamma
\end{array}\right] p_{3} \exp (i \rho x)+\left[\begin{array}{c}
1 \\
-\gamma
\end{array}\right] q_{3} \exp (-i \rho x)
$$

$\phi_{4}=$ Spinor in region IV

$$
=\left[\begin{array}{c}
1 \\
\gamma^{\prime}
\end{array}\right] p_{4} \exp \left(i \rho^{\prime} x\right)+\left[\begin{array}{c}
1 \\
-\gamma^{\prime}
\end{array}\right] q_{4} \exp \left(-i \rho^{\prime} x\right)
$$

$\phi_{5}=$ Spinor in region $\mathrm{V}$

$$
=\left[\begin{array}{l}
1 \\
\gamma
\end{array}\right] p_{5} \exp (i \rho x)+\left[\begin{array}{c}
1 \\
-\gamma
\end{array}\right] q_{5} \exp (-i \rho x)
$$

where

$$
\begin{gathered}
\rho^{2}=\frac{\epsilon\left(\epsilon+2 m^{2} c^{2}\right)}{c^{2} \hbar^{2}} \\
\gamma=\frac{\epsilon}{c \hbar \rho} \\
\rho^{\prime}=i \xi \\
\xi^{2}=\frac{(V-\epsilon)\left(\epsilon+2 m c^{2}-V\right)}{c^{2} \hbar^{2}} \\
\gamma^{\prime}=i \lambda \\
\lambda=\frac{V-\epsilon}{c \hbar \xi}
\end{gathered}
$$

To ensure the absence of Klein paradox and the sub-barrier energies of electron, we have considered the following inequalities in writing the spinors given in Eqs. (7) - (11).

$$
\epsilon<V<\epsilon+m c^{2}
$$




\section{Relativistic formula for lifetime of relativistic resonant tunneling states}

We allow the electron to be incident at the left end of the first barrier in Fig. 1 along the positive direction of the $x$-axis and get it transmitted beyond the right end of the second barrier. The relativistic transmission coefficient, TR, for the DBS is given (for details calculation of TR, see reference [10]) as below:

$$
T_{R}=\frac{1}{1+(F D)^{2}}
$$

where

$$
\begin{gathered}
F=\frac{\gamma^{2}+\lambda^{2}}{2 \gamma \lambda} \sinh \xi b \\
D=2\left[\cosh \xi b \cos \rho a-\frac{\gamma^{2}-\lambda^{2}}{2 \gamma \lambda} \sinh \xi b \sin \rho a\right]
\end{gathered}
$$

Since the quantity, F, cannot be zero anyway, it appears from Eq. (17) that TR assumes maximum value unity and accordingly resonant tunneling occurs when:

$$
D=0
$$

Eq. (20) is the relativistic condition of the resonant tunneling for the DBS. It is apparent from Eqs. (12) - (15) and (19) that D is a function of $\epsilon$. Therefore, the solution of Eq. (20) gives the energies, $\epsilon_{m}$, in which the relativistic resonant tunneling occurs. Now, the expansion of (FD) to the first order in $\left(\epsilon-\epsilon_{m}\right)$ around the $\epsilon_{m}$ can be written as:

$$
F D=[F D]_{\epsilon=\epsilon_{m}}+\left(\epsilon-\epsilon_{m}\right)\left[\frac{d(F D)}{d \epsilon}\right]_{\epsilon=\epsilon_{m}}
$$

Using Eq. (21) the expression of the relativistic transmission co-efficient, $T_{R}$, can be rewritten as:

$$
T_{R}=\left[1+\left(\frac{\epsilon-\epsilon_{m}}{\Delta \epsilon_{D B}}\right)\right]^{-1}
$$

where 


$$
\Delta \epsilon_{D B}=\left[F \frac{d D}{d \epsilon}\right]_{\epsilon=\epsilon_{m}}^{-1}
$$

As can be seen from Eq. (22), the shape of the transmission spectrum is Lorentizian near the energies $\epsilon_{m}$ with $\Delta \epsilon_{D B}$ as half width at half maximum of the transmission peak of $T_{R}$ at $\epsilon_{m}$. The explicit expression of the half width DB can be obtained from Eqs. (12) - (15), (18), (19) and (23) and it reads as:

$$
\Delta \epsilon_{D B}=\left|[F(K+L+M)]_{\epsilon=\epsilon_{m}}^{-1}\right|
$$

where

$$
\begin{aligned}
& K=-\frac{1}{\hbar c}\left[K_{1}+K_{2}\right] \\
& K_{1}=b\left[\left(\frac{\epsilon+2 m c^{2}-V}{V-\epsilon}\right)^{\frac{1}{2}}-\left(\frac{V-\epsilon}{\epsilon+2 m c^{2}-V}\right)^{\frac{1}{2}}\right] \sinh \xi b \cos \rho a \\
& K_{2}=a\left[\left(\frac{\epsilon+2 m c^{2}}{\epsilon}\right)^{\frac{1}{2}}+\left(\frac{\epsilon}{\epsilon+2 m c^{2}}\right)^{\frac{1}{2}}\right] \cosh \xi b \sin \rho a \\
& L=-\frac{\sinh \xi b \sin \rho a}{\epsilon(V-\epsilon)\left(\epsilon+2 m c^{2}\right)\left(\epsilon+2 m c^{2}-V\right)}\left[L_{1}+L_{2}\left(L_{3}-L_{4}+L_{5}+L_{6}\right)\right] \\
& L_{1}=2\left(2 \epsilon-V+2 m c^{2}\right)\left[\epsilon(V-\epsilon)\left(\epsilon+2 m c^{2}\right)\left(\epsilon+2 m c^{2}-V\right)\right]^{\frac{1}{2}} \\
& L_{2}=\epsilon V-\epsilon^{2}-2 m c^{2}+m c^{2} V \\
& L_{3}=\left[\frac{(V-\epsilon)\left(\epsilon+2 m c^{2}\right)\left(\epsilon+2 m c^{2}-V\right)}{\epsilon}\right]^{\frac{1}{2}} \\
& L_{4}=\left[\frac{\epsilon\left(\epsilon+2 m c^{2}\right)\left(\epsilon+2 m c^{2}-V\right)}{(V-\epsilon)}\right]^{\frac{1}{2}}
\end{aligned}
$$




$$
\begin{aligned}
& L_{5}=\left[\frac{\epsilon(V-\epsilon)\left(\epsilon+2 m c^{2}-V\right)}{\left(\epsilon+2 m c^{2}\right)}\right]^{\frac{1}{2}} \\
& L_{6}=\left[\frac{\epsilon(V-\epsilon)\left(\epsilon+2 m c^{2}\right)}{\left(\epsilon+2 m c^{2}\right)}\right]^{\frac{1}{2}} \\
& M=\left[\frac{\epsilon V-\epsilon^{2}-2 m c^{2}+m c^{2} V}{\hbar c\left(\epsilon(V-\epsilon)\left(\epsilon+2 m c^{2}\right)\left(\epsilon+2 m c^{2}-V\right)\right)^{\frac{1}{2}}}\right]\left(M_{1}-M_{2}\right) \\
& M_{1}=b\left[\left(\frac{\epsilon+2 m c^{2}-V}{V-\epsilon}\right)^{\frac{1}{2}}-\left(\frac{V-\epsilon}{\epsilon+2 m c^{2}-V}\right)^{\frac{1}{2}}\right] \cosh \xi b \sin \rho a \\
& M_{2}=a\left[\left(\frac{\epsilon+2 m c^{2}}{\epsilon}\right)^{\frac{1}{2}}+\left(\frac{\epsilon}{\epsilon+2 m c^{2}}\right)^{\frac{1}{2}}\right] \sinh \xi b \cos \rho a
\end{aligned}
$$

It has been shown earlier that the Lorentzian half width of the transmission peak can be related to the lifetime for tunneling $[1,2,5]$. Thus with the help of $\Delta \epsilon_{D B}$ given by Eq. (24), the lifetime of the relativistic resonant tunneling states can be expressed as given below:

$$
\tau_{R}=\frac{\hbar}{2 \Delta \epsilon_{D B}}
$$

\section{Non-relativistic resonant tunneling through DBS}

Our main objective of the present study is to compare critically the relativistic results with NR ones and to elucidate the quantitative relativistic impacts over NR treatment. For this purpose, we intend to produce the NR results pertaining to the resonant tunneling through DBS. Without any ab-initio calculation the expression of NR transmission coefficient and resonant tunneling lifetime can be obtained by subjecting the corresponding relativistic expressions to the condition $c \rightarrow \infty$. In this way we can get the following important NR results.

(i) When the Eq. (17) is subjected to the condition $c \rightarrow \infty$, we get the NR transmission coefficient for DBS as below.

$$
T=\frac{1}{1+(G H)^{2}}
$$


where

$$
\begin{gathered}
G=\frac{k_{1}^{2}+k_{2}^{2}}{2 k_{1} k_{2}} \sinh k_{2} b \\
H=2\left[\cosh k_{2} b \cos k_{1} a-\frac{k_{1}^{2}-k_{2}^{2}}{2 k_{1} k_{2}} \sinh k_{2} b \sin k_{1} a\right]
\end{gathered}
$$

$k_{1}^{2}=\frac{2 m E}{\hbar^{2}}$

$k_{2}^{2}=\frac{2 m(V-E)}{\hbar^{2}}, E<V$

$E$ is NR energy eigenvalue. From Eq. (26) it appears that condition of resonant tunneling is

$$
H=0
$$

because, $G$ cannot be zero anyway. The solution of Eq. (29) gives the energies, $E_{m}$, in which the NR resonant tunneling occurs [4, 12].

(ii)The NR resonant tunneling lifetime can be obtained by subjecting Eq. (25) to the condition $c \rightarrow \infty$ as given below.

$$
\tau=\frac{\hbar}{2 \Delta E_{D B}}
$$

Where $\Delta E_{D B}$ is the half width of Lorentzian NR transmission spectrum at $E_{m}$ and it takes the following form [5]

$$
\Delta E_{D B}=\left|[G(A+B+C)]_{E=E_{m}}^{-1}\right|
$$

where

$$
\begin{aligned}
& A=-\frac{(2 m)^{\frac{1}{2}}}{\hbar}\left[\left(\frac{a}{E^{\frac{1}{2}}}\right) \cosh k_{2} b \sin k_{1} a+\left(\frac{b}{(V-E)^{\frac{1}{2}}}\right) \sinh k_{2} b \cos k_{1} a\right] \\
& B=-\frac{\sinh k_{2} b \sin k_{1} a}{E(V-E)}\left[2(E(V-E))^{\frac{1}{2}}+\frac{1}{2}(V-2 E)\left(\frac{(V-E)^{\frac{1}{2}}}{E^{\frac{1}{2}}}-\frac{E^{\frac{1}{2}}}{(V-E)^{\frac{1}{2}}}\right)\right] \\
& C=-\frac{(V-2 E) m^{\frac{1}{2}}}{\hbar(2 E(V-E))^{\frac{1}{2}}}\left[\frac{b}{(V-E)^{\frac{1}{2}}} \cosh k_{2} b \sin k_{1} a-\frac{a}{E^{\frac{1}{2}}} \sinh k_{2} b \cos k_{1} a\right]
\end{aligned}
$$


Table 1: The data in this table show the values of $E_{m}, \epsilon m$, and $\delta E_{m}$. All data correspond to $\mathrm{V}=50 \mathrm{ev}, \mathrm{a}=5 \AA, \mathrm{b}=0.2 \AA$

\begin{tabular}{|c|c|c|}
\hline \hline $\begin{array}{c}E_{m} \\
\mathrm{ev}\end{array}$ & $\begin{array}{c}\epsilon m \\
\mathrm{ev}\end{array}$ & $\begin{array}{c}\delta E_{m} \\
\mathrm{ev}\end{array}$ \\
\hline 1.094533048974 & 1.094532999899 & $1.19209010^{-7}$ \\
\hline 4.482342999998 & 4.482335997594 & $7.15255710^{-6}$ \\
\hline 10.358781000000 & 10.358707357310 & $7.34329210^{-5}$ \\
\hline 18.867545999980 & 18.867259998390 & $2.86102310^{-4}$ \\
\hline 30.083987999970 & 30.083206999150 & $7.82012910^{-4}$ \\
\hline 44.043879999950 & 44.042141996910 & $1.73950210^{-3}$ \\
\hline \hline
\end{tabular}

\section{Numerical analysis, results and discussion}

The objective behind our numerical analysis is to elucidate quantitatively the circumstances under which the relativistic energies and lifetimes corresponding to the resonant tunneling states differ significantly from NR ones. For this purpose, we investigate the following issues.

Relativistic impact on energies at resonant tunneling: To show this impact we have computed the entity, $\delta E_{m}$, defined as

$\delta E_{m}=E_{m}-\epsilon_{m}$

The NR resonance energies, $E_{m}$, are obtained by solving the condition of resonant tunneling given by Eq. (29). Similarly, the relativistic resonance energies, $\epsilon_{m}$, are determined from the relativistic condition given by Eq. (20). The results about Em are shown in Table - 1 and in Fig. 2.

Relativistic impact on resonant tunneling lifetime: To see the quantitative impact on relativistic resonant tunneling lifetime, we have computed $\tau$ and $\tau_{R}$ from Eqs. (30) and (25) respectively. We plot $\tau$ and $\tau_{R}$. It is shown in Fig. 3. The parameters taken here are akin to what have been used by others $[1,3,11]$ in the numerical analysis of the problem related to resonant tunneling through DBS.

In Table - 1, we see that the energies $\left(\epsilon_{m}\right)$ at which relativistic resonant tunneling occurs decreases with respect to the energies $\left(E_{m}\right)$ of NR resonant tunneling. This table also shows that in the range of low energies, the relativistic impact estimated by $\delta E_{m}$ assumes small values and it becomes appreciably large in the range of high energy. The graph in Fig.2 depicts clearly how the relativistic impact, $\delta E_{m}$, varies with $E_{m}$. It can be noticed that $\delta E_{m}$ increases with $E_{m}$ and tends to become more or less constant at higher values of $E_{m}$. Looking at the graphs in Fig.3 we have the following prominent features:

(i) Both $\tau$ and $\tau_{R}$ decreases with increase of resonance energies; the profiles corresponding to this decrement are nearly exponential. $\tau$ and $\tau_{R}$ both are inversely proportional to the half width at half maximum of the transmission peak as shown in the Eqs. (30) and (25) respectively. Therefore, this situation suggests that the 


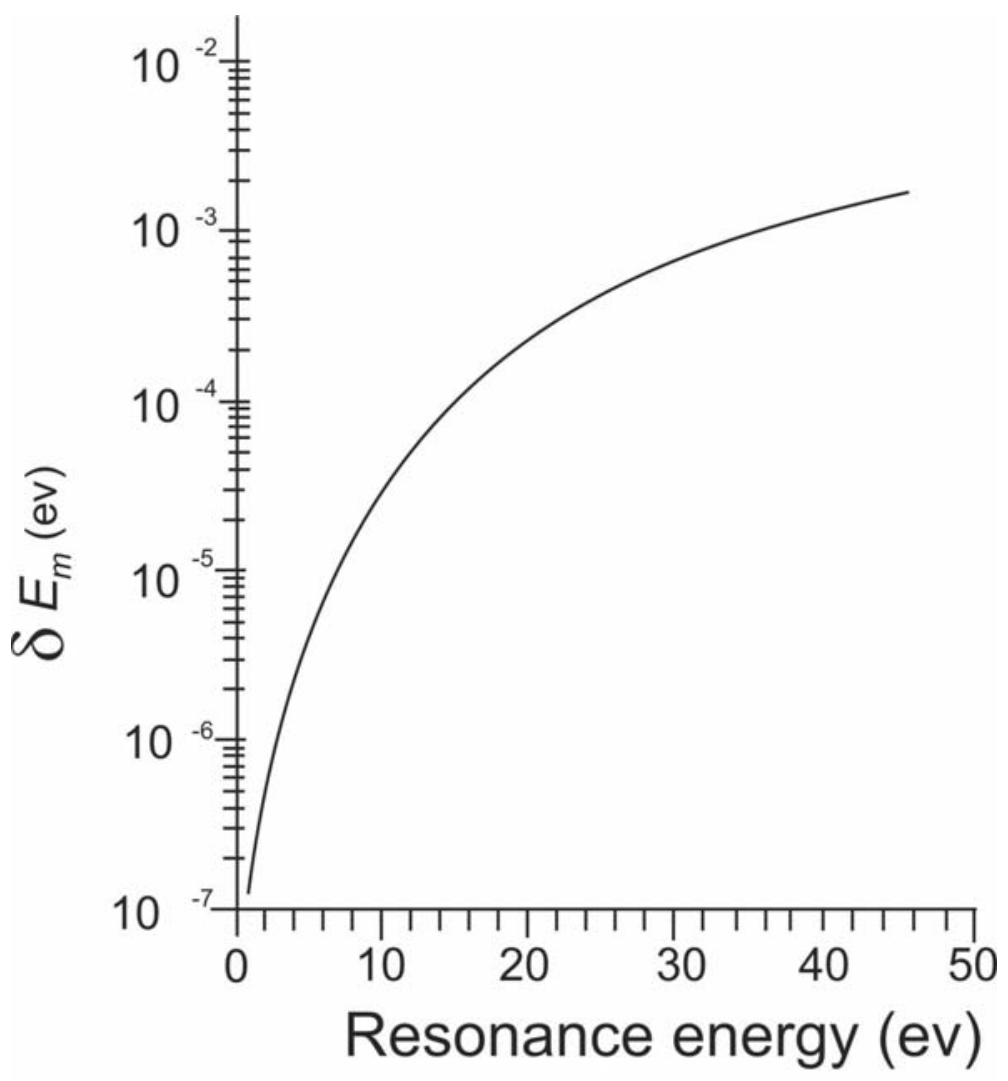

Figure 2: (a)Variation of $\delta E_{m}$ with $E_{m}$. The values of parameters are as follows: $V$ $=50 \mathrm{ev}, \mathrm{a}=5 \stackrel{\circ}{\AA}, \mathrm{b}=0.2 \stackrel{\circ}{A}$.

peaks of spectrum of both $T$ and $T_{R}$ become wider at the larger resonance energies.

(ii) The relativistic treatment reveals the most important and prominent feature that $\tau_{R}$ always assumes smaller value as compared to $\tau$. This aspect also confirms a wider peak of relativistic transmission spectrum for any value of resonance energy as compared to NR resonance.

To the end, we can conclude that our present work shows an appreciable relativistic impact on resonant tunneling. The technological implication of the results reported in this paper is that the extent of the relativistic effect on the resonant tunneling lifetime would become important in the studies of solid state devices involving with electron transmission through DBS because of the following reasons:

(i) It has been found that $\tau_{R}$ always assumes smaller value compared to $\tau$. It means that under relativistic treatment the phase velocity of the electron corresponds to a greater value. The present treatment shows an appreciable relativistic impact that corresponds to smaller values of $\tau_{R}$ as compared to $\tau$. This feature appears to be helpful in the analysis and fabrication of very high speed solid state devices.

(ii) The current density through resonant tunneling in DBS is related to the half width at half maximum of the transmission peak and it suggests that the wider the 


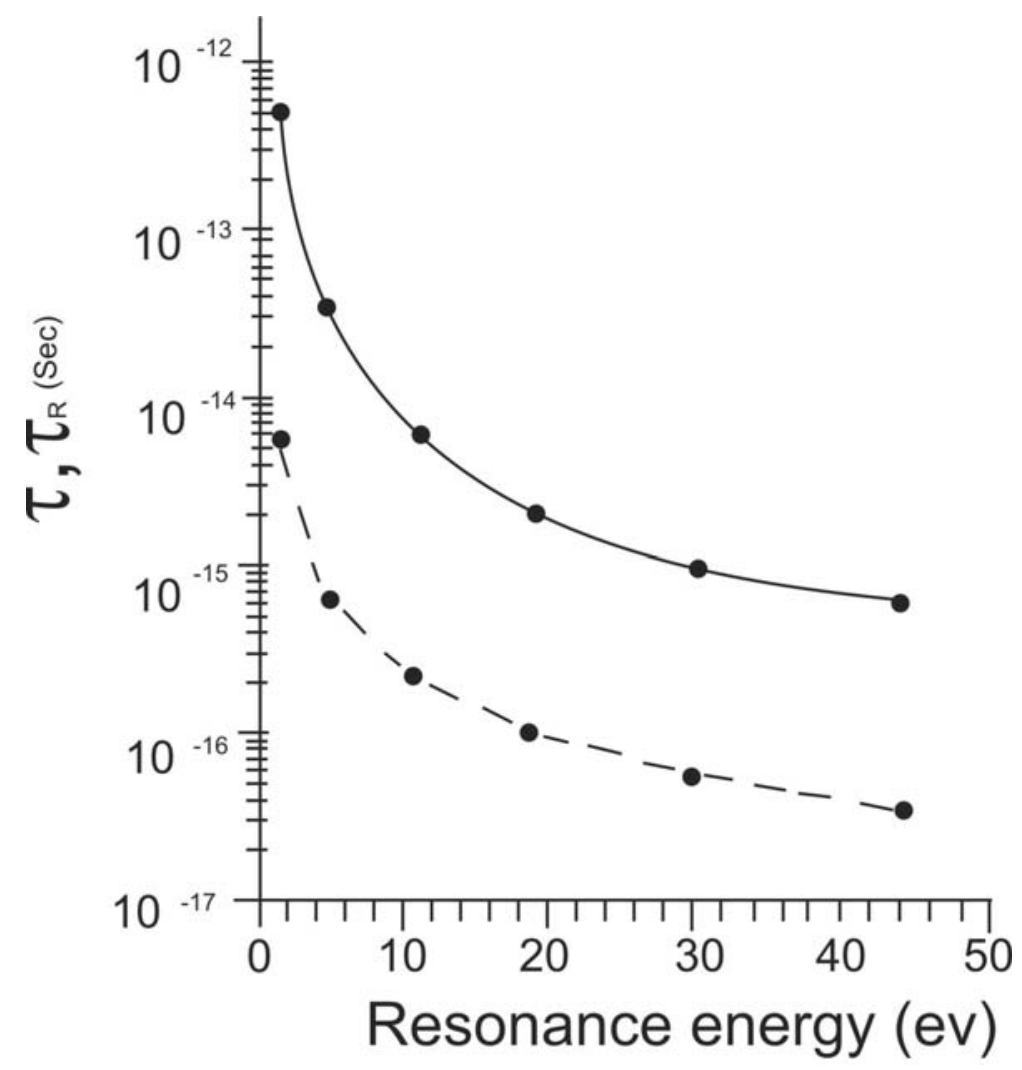

Figure 3: Variation $\tau$ and $\tau_{R}$ of with respect to resonance energy. The values of parameters are as follows: $V=50 \mathrm{ev}, \mathrm{a}=5 \stackrel{\circ}{A} \mathrm{~b}=0.2 \stackrel{\circ}{A}$.

peak in the transmission coefficient, the larger the current density is $[1,5,6]$. Our findings that the relativistic transmission spectrum around the resonance energies is always wider than the corresponding NR transmission spectrum confirm a greater total current transmitted via relativistic resonant tunneling. This fact would be very useful to study and engineer any resonant tunneling devices.

\section{References}

[1] C. J. Arsenault and M. Meunier, Resonant-tunneling lifetime comparison between double-barrier and $\delta$-doped barrier structures, Phys. Rev B,39 (1989), $8739-8742$.

[2] P. J. Price, Theory of resonant tunneling in heterostructures, Phys. Rev B, 38 (1988), 1994 - 1998.

[3] H. Xu, M. Zhu, B. Hou, Analytical expressions for resonant tunneling lifetime in symmetrical double-barrier structures, Phys. Lett A, 223 (1996), 227 - 231. 
[4] C. L. Roy and A. Khan, Some typical features of resonant tunneling in multibarrier systems, Phys. Stat Solidi b, 176 (1993), K47 - K49.

[5] A. Khan, P. K. Mahapatra and C. L. Roy, Lifetime for resonant tunneling in multibarrier systems, Phys. Lett A, 249(1998), 512 - 516.

[6] A. Khan, P. K. Mahapatra, S. P. Bhattacharya, and S. N. Mohammad, Resonant tunneling lifetime in semiconductor superlattice, Phil Mag, 84 (2004), 547 -563 .

[7] P. Panchadhyayee, R. Biswas, A. Khan, and P. K. Mahapatra, Electric-fieldinduced resonant tunneling lifetime in semiconductor multibarrier systems, $J$. App Phys, 104 (2008), 084517-1 - 084517-4.

[8] P. Pereyra, Closed formulas for tunneling time in superlattices, Phys. Rev. Lett, 84 (2000), $1772-1775$.

[9] P. K. Mahapatra, P. Panchadhyayee, S. P. Bhattacharya, and A. Khan, Resonant tunneling in electrically biased multibarrier system, Physica B, 403 (2008), $2780-2788$.

[10] C L Roy and A. Khan, A relativistic study of tunneling through double barriers, Indian J. Pure and Appl. Phys, 30(1992), 432 - 438.

[11] C L Roy and A. Khan, Relativistic impacts on tunneling through multibarrier systems, J. Phys: Condens Matter, 5 (1993), 7701 - 7708.

[12] C. L. Roy and A. Khan, Some features of resonant tunneling, J. Phys Chem Solid, 53 (1992), 383 - 385.

Received: March, 2012 